\title{
Chrysotile fibers in tissue adjacent to laryngeal squamous cell carcinoma in cases with a history of occupational asbestos exposure
}

\author{
Stephanie K. Wronkiewicz ${ }^{1}$ - Victor L. Roggli ${ }^{2}$ - Benjamin H. Hinrichs ${ }^{3}$ - Ady Kendler ${ }^{3}$ - Rondi A. Butler ${ }^{4}$. \\ Brock C. Christensen ${ }^{5,6}$. Carmen J. Marsit ${ }^{7,8}$ - Heather H. Nelson ${ }^{9,10}$ • Michael D. McClean ${ }^{11} \cdot$ Karl T. Kelsey $^{4,12}$. \\ Scott M. Langevin (iD) ${ }^{13,14}$
}

Received: 25 March 2019 / Revised: 9 May 2019 / Accepted: 3 July 2019 / Published online: 5 August 2019

(c) United States \& Canadian Academy of Pathology 2019

\begin{abstract}
Asbestos describes a group of naturally occurring fibrous silicate mineral compounds that have been associated with a number of respiratory maladies, including mesothelioma and lung cancer. In addition, based primarily on epidemiologic studies, asbestos has been implicated as a risk factor for laryngeal and pharyngeal squamous cell carcinoma (SCC). The main objective of this work was to strengthen existing evidence via empirical demonstration of persistent asbestos fibers embedded in the tissue surrounding laryngeal and pharyngeal SCC, thus providing a more definitive biological link between exposure and disease. Six human papillomavirus (HPV)-negative laryngeal $(n=4)$ and pharyngeal $(n=2)$ SCC cases with a history working in an asbestos-exposed occupation were selected from a large population-based case-control study of head and neck cancer. A laryngeal SCC case with no history of occupational asbestos exposure was included as a control. Tissue cores were obtained from adjacent nonneoplastic tissue in tumor blocks from the initial primary tumor resection, and mineral fiber analysis was performed using a scanning electron microscope equipped with an energy dispersive X-ray analyzer (EDXA). Chrysotile asbestos fiber bundles were identified in 3/6 of evaluated cases with a history of occupational asbestos exposure. All three cases had tumors originating in the larynx. In addition, a wollastonite fiber of unclear significance was identified one of the HPV-negative pharyngeal SCC cases. No mineral fibers were identified in adjacent tissue of the case without occupational exposure. The presence of asbestos fibers in the epithelial tissue surrounding laryngeal SCC in cases with a history of occupational asbestos exposure adds a key line of physical evidence implicating asbestos as an etiologic factor.
\end{abstract}

Karl T. Kelsey

karl_kelsey@brown.edu

$\triangle$ Scott M. Langevin

langevst@ucmail.uc.edu

1 McMicken College of Arts and Sciences, University of Cincinnati, Cincinnati, OH, USA

2 Department of Pathology, Duke University School of Medicine, Durham, NC, USA

3 Department of Pathology and Laboratory Medicine, University of Cincinnati, College of Medicine, Cincinnati, OH, USA

4 Department of Epidemiology, Brown University School of Public Health, Providence, RI, USA

5 Department of Epidemiology, Geisel School of Medicine at Dartmouth, Lebanon, NH, USA

6 Department of Molecular and Systems Biology, Geisel School of
Medicine at Dartmouth, Lebanon, NH, USA

7 Department of Environmental Health, Rollins School of Public Health, Emory University, Atlanta, GA, USA

8 Winship Cancer Institute, Emory University, Atlanta, GA, USA

9 Masonic Cancer Center, Minneapolis, MN, USA

10 Division of Epidemiology and Community Health, University of Minnesota School of Public Health, Minneapolis, MN, USA

11 Department of Environmental Health, Boston University School of Public Health, Boston, MA, USA

12 Department of Pathology \& Laboratory Medicine, Alpert Medical School, Brown University, Providence, RI, USA

13 Division of Epidemiology, Department of Environmental Health, University of Cincinnati, College of Medicine, Cincinnati, OH, USA

14 Cincinnati Cancer Center, Cincinnati, OH, USA 


\section{Introduction}

According to the most recent global cancer statistics, pharyngeal and laryngeal cancer accounted for an estimated 350,917 global incident cancer diagnoses and 180,770 deaths in 2018 [1]. Of these, more than $90 \%$ are squamous cell carcinoma (SCC) [2], with smoking, alcohol consumption, and human papillomavirus (HPV) infection, particularly for oropharyngeal SCC, being widely recognized as the primary risk factors [3]. While those risk factors have garnered the majority of attention in etiologic research - deservingly so since they are attributed to the vast majority of these tumors-there is evidence that occupational exposures are also risk factors $[4,5]$.

Asbestos describes a group of naturally occurring fibrous silicate mineral compounds. Its use dates back thousands of years, but became widespread in the late 19th century through the late 20th century [6] due to its favorable industrial properties, including its strength, flexibility, and thermal properties, with peak use in the United States occurring in the 1970s. Asbestos has been associated with a number of chronic respiratory diseases [7], including malignancy, and was linked to mesothelioma $[8,9]$ and lung cancer [10] in the early to mid-20th century. Despite the known health risks, asbestos is not universally banned, and is still in use in many countries (including the United States), with an estimated 125 million occupationally exposed individuals worldwide [11].

In addition to mesothelioma and lung cancer, asbestos has also been implicated as a risk factor for cancers involving the upper airway, in particular laryngeal and pharyngeal SCC [4, 12-14]; however, the evidence thus far has been primarily based on epidemiologic data from observational studies. Therefore, the goal of this study was to strengthen the existing evidence implicating asbestos as a risk factor for pharyngeal and/or laryngeal SCC via confirmation of persistent asbestos fibers in epithelial tissue surrounding laryngeal and pharyngeal SCC in cases with a history of occupational asbestos exposure.

\section{Materials and methods}

\section{Study population}

Six cases with incident, HPV-negative pharyngeal $(n=2)$ and laryngeal $(n=4)$ SCC (ICD-9: 146, 148, 149.0, 149.1, and 161) with self-reported occupational exposure to asbestos were selected from a large population-based case-control study of head and neck cancer, plus one laryngeal SCC case with no occupational exposure history as a control, matched to the sex, race/ethnicity, and median age ( \pm 3 years) and smoking pack-years $( \pm 3$ pack-years) of the asbestos-exposed cases. Briefly, detailed occupational history was available for 1056 head and neck cancer cases enrolled through major teaching hospitals located in Boston, Massachusetts [15-17]. Occupational history was systematically reviewed to verify that cases with self-reported occupational history worked in an occupation at high risk for asbestos exposure. All cases selected had archival tumor and adjacent normal tissue available at Massachusetts General Hospital.

\section{High-risk HPV testing}

Serologic testing was performed for antibodies against E6 and E7 viral oncoproteins for high-risk HPV (HPV16, 18, $31,33,45,52$, and 58) using sandwich ELISA assays, as previously described [18-22]. Subjects were considered high-risk HPV seropositive if antibodies were present for either viral oncoprotein.

\section{Asbestos fiber counts}

Cores were obtained from adjacent nonneoplastic tissue in tumor blocks from the initial primary tumor resection. A count of asbestos fibers $\geq 5 \mu \mathrm{m}$ was performed on the cores from each case at Duke University via scanning electron microscopy [23]. Specimens were deparaffinized in xylene, rehydrated in $95 \%$ ethanol, and wet weight was obtained on an analytical balance. The tissue was then digested in sodium hypochlorite solution, and the residue collected on $13-\mathrm{mm}$ Nuclepore filters with a pore size of $0.4 \mu \mathrm{m}$. These were mounted on a carbon stub with colloidal graphite, sputter-coated with platinum, and examined by scanning electron microscopy (SEM) equipped with an energy dispersive X-ray analyzer (EDXA). One hundred consecutive fields were examined at a screen magnification of $\times 1300$ and all fibers $\geq 5 \mu \mathrm{m}$ in length counted, and examined by EDXA [23].

\section{Immune assessment}

For descriptive purposes, morphologic analysis was performed to assess severity and type of inflammation in the tumor microenvironment. "Global" qualitative assessments of inflammation severity were performed by two boardcertified Anatomic Pathologists (each blinded to the other's assessments), as well as assessments of the presence or absence of dense lymphoid tissue and neutrophilic inflammation. The lymphoid infiltrate in each case was assigned one of three "patterns" depending on whether lymphoid tissue around tumor cells formed a continuous and dense 
rim (Pattern 1), was present as discontinuous dense patches (Pattern 2), or was limited or absent (Pattern 3) [24]. Neutrophilic inflammation and global qualitative inflammation assessments were also scored as three-tiered variables $(0-25 \%, 25-50 \%,>50 \%)$ and as mild, moderate, and severe, respectively [25].

\section{Results}

Study subject characteristics, including occupation(s) and duration spent working in an occupation with asbestos exposure, are presented in Table 1. Briefly, all asbestosexposed cases were Caucasian males with a median age of 63.5 years (range: 56-77 years). Asbestos-exposed occupations/industries of the cases included automotive mechanic, space shuttle thermal protection applicator (solidfuel boosters contained asbestos materials [26]), firefighter, Army artillery, school custodian, and metal lather, with a median duration spent in an asbestos-exposed occupation of 34.5 years (range: 5-56 years). The non-asbestos-exposed case was a 64-year-old Caucasian male with an occupational history as a truck driver. With the exception of one of the cases (HN2), all subjects had a smoking history, with a median of 39 packs-years (range: 0-114 pack-years). The reported occupational asbestos exposures for all cases overlapped peak industrial usage in the United States [27] as depicted in Fig. 1.

Histopathologic slides were available for the primary tumors of $4 / 6$ cases (Fig. 2). Of those, two distinct patterns of inflammation were noted. Two cases showed prominent lymphoid infiltrates, which were present as a continuous dense rim in one case (HN1) and as discontinuous dense patches in the other (HN6), with each exhibiting minimal to absent neutrophilic inflammation. In contradistinction, the other two cases (HN3 and HN4) contained limited or no lymphoid tissue, but did show prominent neutrophilic inflammation.

Chrysotile asbestos fiber bundles were identified by SEM in adjacent nonneoplastic tissue from 3/6 asbestos-exposed cases with spectra confirmed by EDXA, all of which had laryngeal SCC (Fig. 3); neither of the two HPV-negative pharyngeal cancer cases had detectable asbestos fibers. Fibers ranged from 7 to $14 \mu \mathrm{m}$ in length and 0.2 to $0.5 \mu \mathrm{m}$ in diameter. It should be noted that, given the archival nature of the tissue, samples for all cases were well below the recommended amount of $0.3 \mathrm{~g}$ wet weight for fiber analysis (Table 2). A wollasonite fiber (calcium silicate) was identified in adjacent tissue in one of the two HPV-negative oropharyngeal cases (HN4), although this is of uncertain significance. No mineral fibers were identified in the adjacent tissue of the case without any known occupational exposure (control).

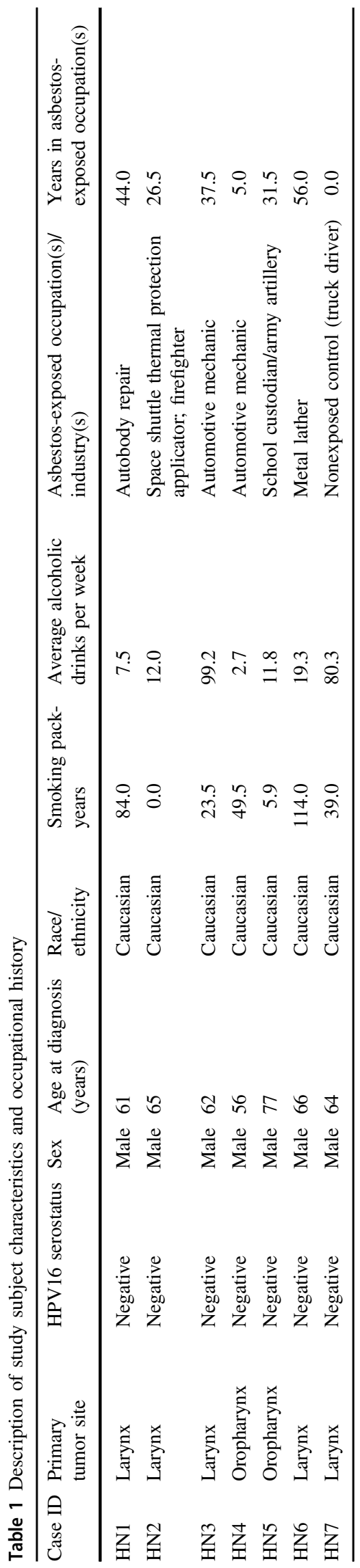




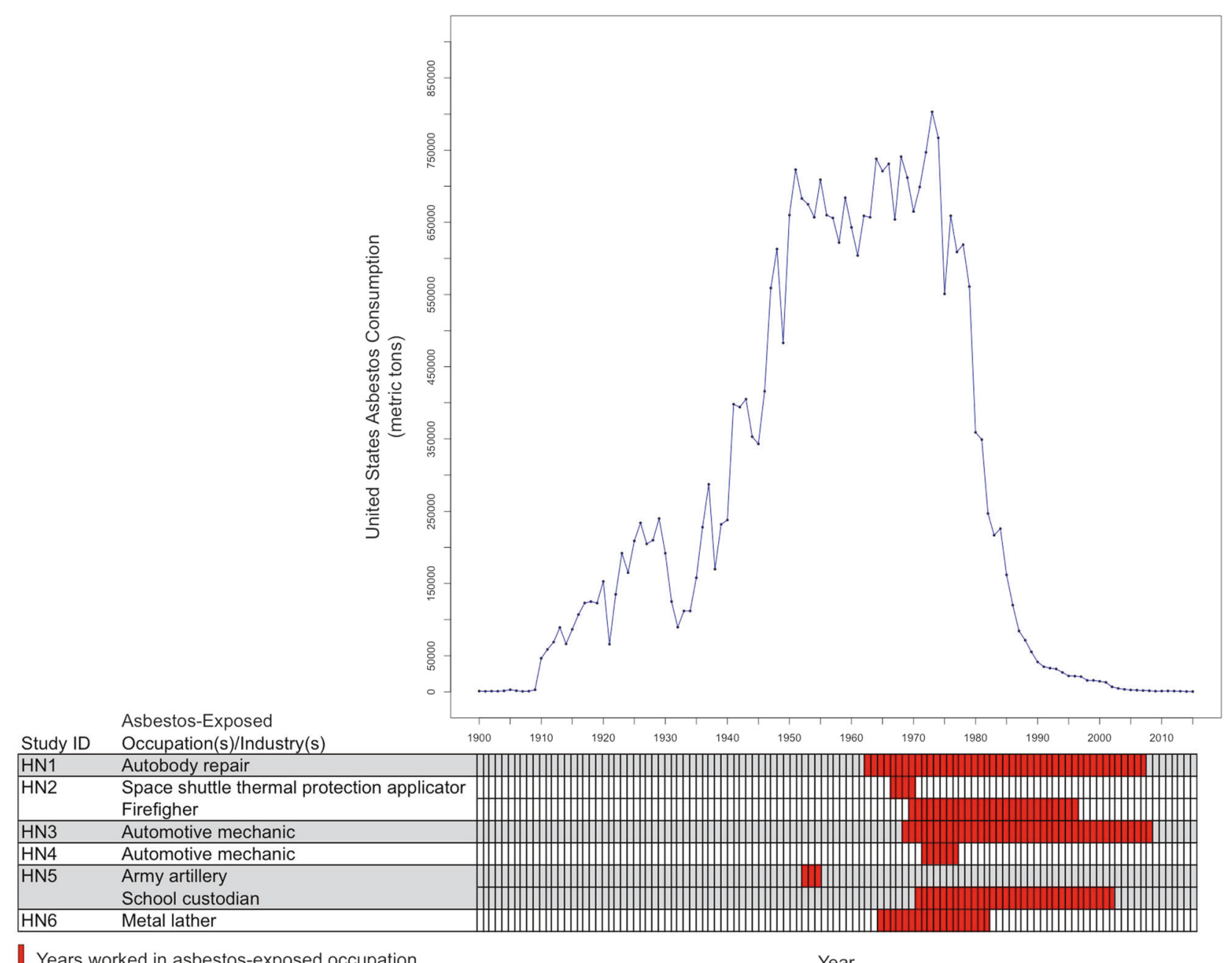

Fig. 1 Timeframe of occupational asbestos exposure for cases in the context of annual industrial asbestos consumption in the United States. The rows at the bottom of the figure correspond to the occupation with

\section{Discussion}

We have identified chrysotile asbestos fibers in nonneoplastic epithelial tissue adjacent to laryngeal SCC in cases with a history of work in an asbestos-exposed occupation, greatly strengthening the causal evidence for asbestos as a risk factor. Up to now, there had only been one other case report identifying mostly amphibole asbestos fibers in nonneoplastic laryngeal tissue in a case with laryngeal SCC [28]. This work significantly adds to these previous reports, quadrupling the number of reported laryngeal SCC cases with detectable asbestos fibers and, importantly represents the first study to identify chrysotile asbestos fibers proximal to laryngeal SCC tumors in the absence of amphibole fibers.

These findings are in agreement with and complementary to existing epidemiological evidence from industrial cohorts and observational studies supporting an association of asbestos exposure for the cases, where red boxes represent years working in each occupation. The upper plot shows annual asbestos consumption in the United States from 1900 to 2015

asbestos exposure and laryngeal SCC [12, 14]. Our observations are also in-line with that from two separate reports of asbestos bodies and/or fibers in laryngeal tissue at autopsy from former asbestos workers with no detectable laryngeal cancer $[28,29]$. Of particular interest in our present study is the identification of chrysotile fibers in tissue from a laryngeal SCC case who identified as a never smoker and moderate alcohol user-hardly the prototypical profile of a laryngeal cancer case-but with a high-risk occupational history working as a space shuttle thermal protection system applicator in the 1970s and over two decades as a firefighter (HN2).

Chrysotile fibers were only identified in asbestosexposed laryngeal SCC cases, with none identified in tissue from either HPV-negative pharyngeal SCC case. However, it bears to be reiterated that the samples in this study ranged 2.7-fold to 3000-fold below the recommended $0.3 \mathrm{~g}$ of wet tissue weight that would be used with larger 


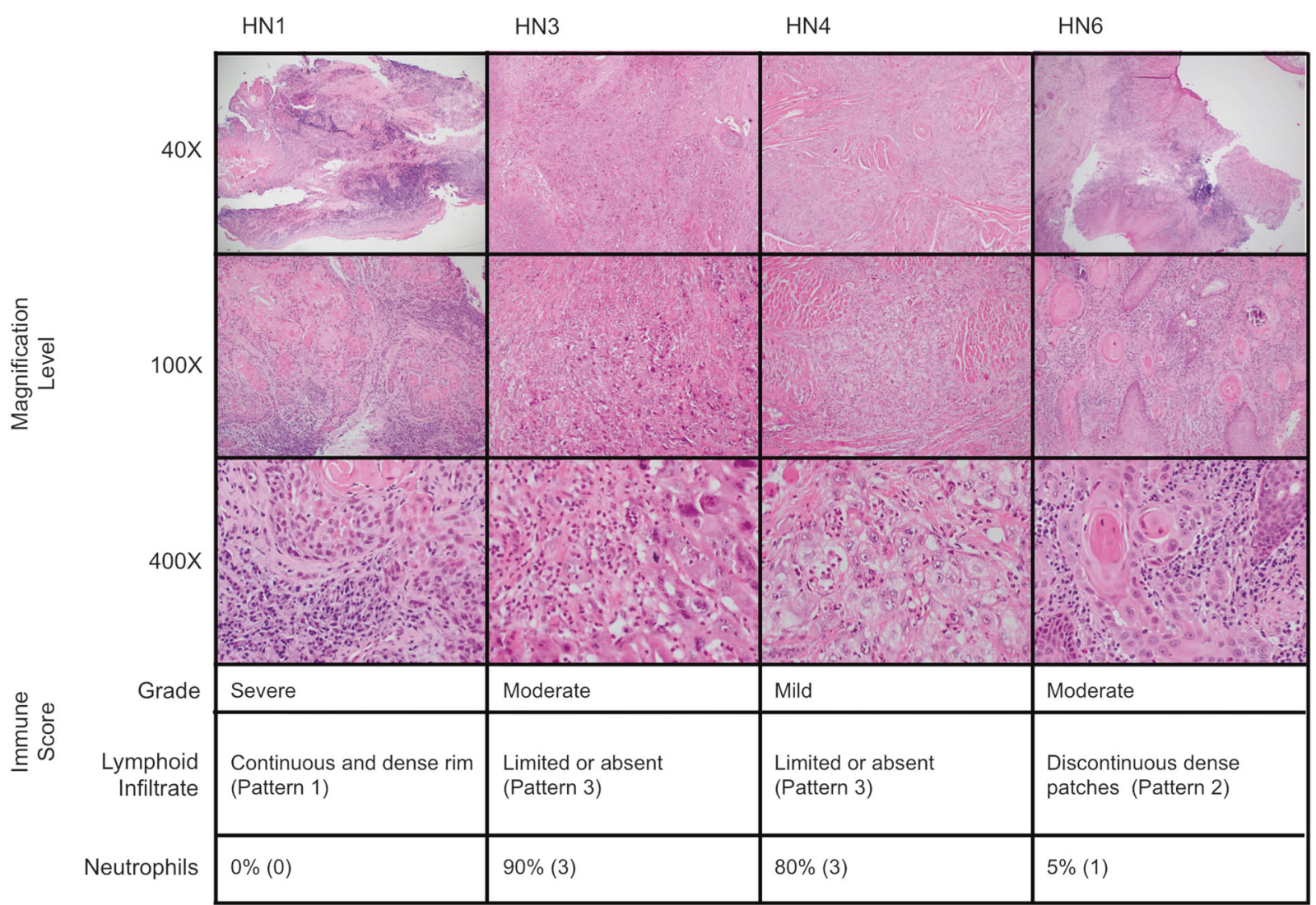

Fig. 2 Representative formalin-fixed-paraffin embedded tumor sections stained with hematoxylin and eosin (H\&E) from cases with an occupational asbestos exposure history and available tumor tissue, with corresponding immune scores

HN2: chrysotile bundle $(\times 11000)$

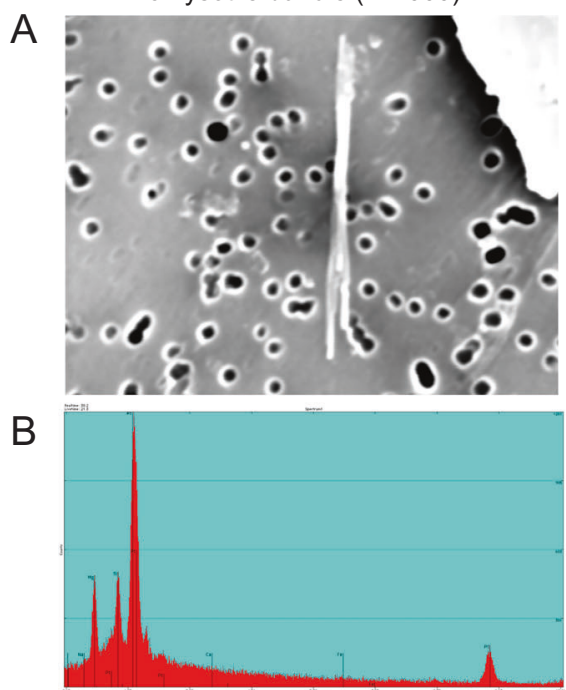

HN3: chrysotile bundle $(\times 7000)$
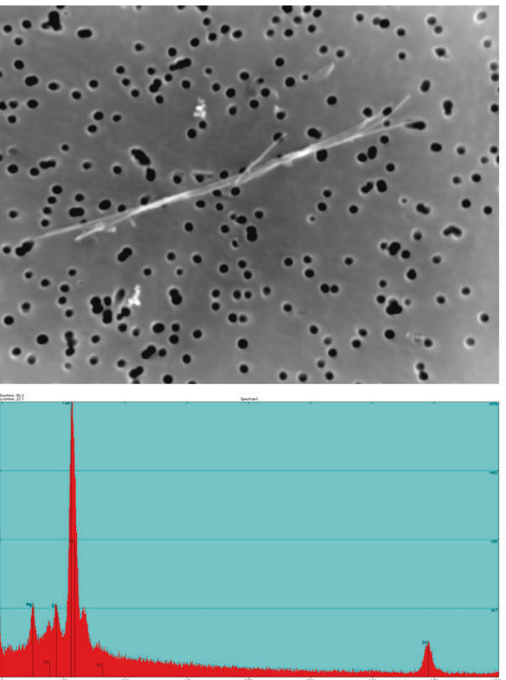

HN6: chrysotile bundle $(\times 7500)$
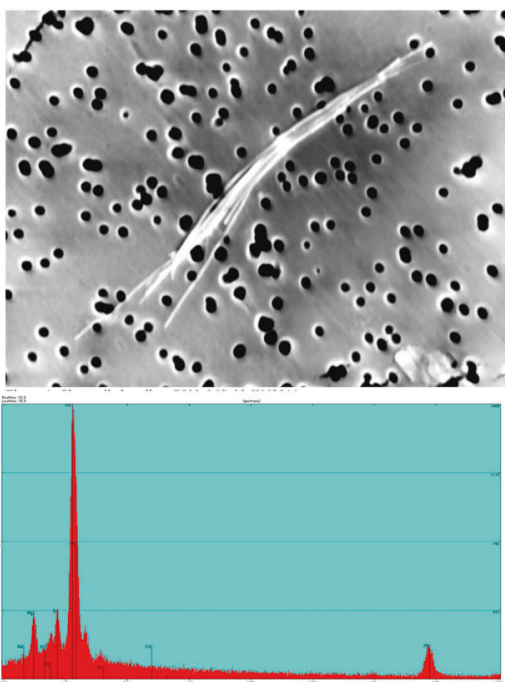

Fig. 3 Analysis of tissue mineral fiber content. a Scanning electron microscopy (SEM) images of chrysotile asbestos fibers identified in tissue adjacent to laryngeal squamous cell carcinoma tumor in three

autopsy samples, and therefore samples with no identifiable asbestos fibers should be considered inconclusive rather than negative. To this point, one of the two pharyngeal cases with a history of occupational asbestos exposure: HN2, HN3, and HN6. b The corresponding energy dispersive X-ray analyzer (EDXA) spectrum consistent with chrysotile asbestos fibers

cases (HN5) had the least amount of tissue available of all of the samples. Wollastonite-an acicular calcium silicate mineral fiber-was identified in the other of the two 
Table 2 Quantity of adjacent tissue for asbestos fiber count analysis

Case ID Number of cores for fiber Sample wet weight ${ }^{\mathrm{a}}(\mathrm{g})$ analysis

\begin{tabular}{lll}
\hline HN1 & (1) $2 \mathrm{~mm}$ core & 0.0076 \\
HN2 & (1) $2 \mathrm{~mm}$ core & 0.0030 \\
HN3 & (3) $2 \mathrm{~mm}$ core & 0.0004 \\
HN4 & (4) $2 \mathrm{~mm}$ core & 0.0110 \\
HN5 & (1) $2 \mathrm{~mm}$ core & 0.0001 \\
HN6 & (3) $2 \mathrm{~mm}$ core & 0.0001 \\
HN7 & (5) $2 \mathrm{~mm}$ core & 0.0020 \\
\hline
\end{tabular}

${ }^{a}$ Measured after deparaffinization and rehydration of tissue

HPV-negative oropharyngeal cases (HN5). However the significance of this is uncertain, as an IARC working group determined two decades ago that wollastonite cannot be classified as to its carcinogenicity to humans due to insufficient human or experimental models [30], and little to no evidence to the contrary has been surfaced since [31].

There were several strengths to our study, notably use of SEM for fiber analysis with confirmation by EDXA spectra; detailed occupational history and other major risk factor data for the cases; and availability of adjacent nonneoplastic epithelial tissue, which allowed us to avoid a dilution effect that would be encountered by using tumor tissue due to clonal expansion. However, there were also some limitations. Due to labor and resource constraints, including availability of archival tissue materials, we were only able to evaluate a small subset of cases with occupational asbestos exposure in this study [17]. However, despite the small sample size, it is notable that chrysotile fibers were identified in $3 / 6$ of the cases interrogated, and were absent from the cases lacking an occupational asbestos history. Moreover, as we had previously stated, the wet tissue weight for each case was suboptimal for fiber detection, and therefore we cannot rule out the presence of fibers in pharyngeal or laryngeal tissue from the negative cases. Nonetheless, we still were able to identify fibers in a sizeable fraction of interrogated cases.

Despite its known adverse health effects, asbestos continues to be a threat to global health. Although asbestos has been fully banned more than 50 countries, it is still in widespread use among developing nations, and is notably still in use-albeit limited-in the United States [32, 33]. Our identification of chrysotile asbestos fibers in epithelial tissue proximal laryngeal SCC adds a critical empirical link, and bolsters the mounting evidence that asbestos is a risk factor for laryngeal cancer.

Acknowledgements This work was supported by the NIH/NIDCR grant R21DE027227 to SML; NIH/NCI grant R01CA100679 to KTK; and NIH/NIEHS supported UC Center for Environmental Genetics (2P30ES006096).

\section{Compliance with ethical standards}

Conflict of interest The authors declare that they have no conflict of interest.

Publisher's note: Springer Nature remains neutral with regard to jurisdictional claims in published maps and institutional affiliations.

\section{References}

1. Bray F, Ferlay J, Soerjomataram I, Siegel RL, Torre LA, Jemal A. Global cancer statistics 2018: GLOBOCAN estimates of incidence and mortality worldwide for 36 cancers in 185 countries. CA Cancer J Clin. 2018;68:394-424.

2. Carvalho AL, Nishimoto IN, Califano JA, Kowalski LP. Trends in incidence and prognosis for head and neck cancer in the United States: a site-specific analysis of the SEER database. Int J Cancer. 2005; 114:806-16

3. Marur S, Forastiere AA. Head and neck cancer: changing epidemiology, diagnosis, and treatment. Mayo Clin Proc. 2008;83:489-501.

4. Paget-Bailly S, Cyr D, Luce D. Occupational exposures to asbestos, polycyclic aromatic hydrocarbons and solvents, and cancers of the oral cavity and pharynx: a quantitative literature review. Int Arch Occup Environ Health. 2012;85:341-51.

5. Paget-Bailly S, Cyr D, Luce D. Occupational exposures and cancer of the larynx-systematic review and meta-analysis. J Occup Environ Med. 2012;54:71-84.

6. Weiss SH. A call to action: epidemiologists assert themselves with scientific data. Int J Occup Environ Health. 2012;18:167-70.

7. Jamrozik E, de Klerk N, Musk AW. Asbestos-related disease. Intern Med J. 2011;41:372-80.

8. Gloyne S. Two cases of squamous cell carcinoma of the lung occuring in asbestosis. Tubercle. 1935;17:5-10.

9. Wagner JC, Sleggs CA, Marchand P. Diffuse pleural mesothelioma and asbestos exposure in the North Western Cape Province. Br J Ind Med. 1960;17:260-71.

10. Doll R. Mortality from lung cancer in asbestos workers. Br J Ind Med. 1955;12:81-6.

11. WHO. Asbestos: elimination of asbestos-related diseases. 2010. http://www.who.int/mediacentre/factsheets/fs343/en/index.html. Accessed 28 February 2013.

12. IOM Committee on Asbestos. Asbestos: selected cancers. Institute of Medicine of the National Academies: Washington, DC: The National Academies Press. https://doi.org/10.17226/11665. 2006.

13. Awan KH, Hegde R, Cheever VJ, Carroll W, Khan S, Patil S, et al. Oral and pharyngeal cancer risk associated with occupational carcinogenic substances: systematic review. Head Neck. 2018;40:2724-32.

14. IARC Working Group. Asbestos (chrysotile, amosite, crocidolite, tremolite, actinolyte, and anthophylite). Lyon, France: International Agency for Research on Cancer (IARC); 2012.

15. Fogleman EV, Eliot M, Michaud DS, Nelson HH, McClean MD, Langevin SM, et al. Occupational asphalt is not associated with head and neck cancer. Occup Med. 2015;65:570-3.

16. Langevin SM, McClean MD, Michaud DS, Eliot M, Nelson HH, Kelsey KT. Occupational dust exposure and head and neck squamous cell carcinoma risk in a population-based case-control study conducted in the greater Boston area. Cancer Med. 2013;2:978-86. 
17. Langevin SM, O'Sullivan MH, Valerio JL, Pawlita M, Applebaum KM, Eliot M, et al. Occupational asbestos exposure is associated with pharyngeal squamous cell carcinoma in men from the greater Boston area. Occup Environ Med. 2013;70:858-63.

18. Waterboer T, Sehr P, Michael KM, Franceschi S, Nieland JD, Joos TO, et al. Multiplex human papillomavirus serology based on in situ-purified glutathione s-transferase fusion proteins. Clin Chem. 2005;51:1845-53.

19. Kelsey KT, Nelson HH, Kim S, Pawlita M, Langevin SM, Eliot $\mathrm{M}$, et al. Human papillomavirus serology and tobacco smoking in a community control group. BMC Infect Dis. 2015;15:8.

20. Nelson HH, Pawlita M, Michaud DS, McClean M, Langevin SM, Eliot MN, et al. Immune response to HPV16 E6 and E7 proteins and patient outcomes in head and neck cancer. JAMA Oncol. 2017;3:178-85.

21. Michaud DS, Langevin SM, Eliot M, Nelson HH, Pawlita M, McClean MD, et al. High-risk HPV types and head and neck cancer. Int J Cancer. 2014;135:1653-61.

22. Tan X, Nelson HH, Langevin SM, McClean M, Marsit CJ, Waterboer $\mathrm{T}$, et al. Obesity and head and neck cancer risk and survival by human papillomavirus serology. Cancer Causes Control. 2015;26:111-9.

23. Roggli VL, Sharma A. Analysis of tissue mineral fiber content. In: V.L. R, Oury TD, Sporn TA, editors. Pathology of asbestosassociated diseases. 3rd edn. New York: Springer:; 2014. p. 253-92.

24. Brandwein-Gensler M, Teixeira MS, Lewis CM, Lee B, Rolnitzky L, Hille JJ, et al. Oral squamous cell carcinoma: histologic risk assessment, but not margin status, is strongly predictive of local disease-free and overall survival. Am J Surg Pathol. 2005;29:167-78.

25. Lundqvist L, Stenlund H, Laurell G, Nylander K. The importance of stromal inflammation in squamous cell carcinoma of the tongue. J Oral Pathol Med. 2012;41:379-83.

26. Rogers WP, Armstrong NA, Acheson DC, Covert EE, Feynman RP, Hotz RB, et al. Report of the Presidential Commission on the Space Shuttle Challenger Accident: Washington, DC; 1986.

27. USGS. U.S. Geological Survey, 2017, Asbestos Statistics, [through 2015, last modified January 19, 2017], 2017.

28. Hirsch A, Bignon J, Sebastien P, Gaudichet A. Asbestos fibers in laryngeal tissues. Findings in two patients with asbestosis associated with laryngeal tumors. Chest. 1979;76:697-9.

29. Roggli VL, Greenberg SD, McLarty JL, Hurst GA, Spivey CG, Heiger LR. Asbestos body content of the larnyx in asbestos workers. A study of five cases. Arch Otolaryngol. 1980;106:533-5.

30. IARC Working Group. Silica, some silicates, coal dust and paraaramid fibrils. Lyon, France: International Agency for Research on Cancer (IARC); 1997.

31. Maxim LD, McConnell EE. A review of the toxicology and epidemiology of wollastonite. Inhal Toxicol. 2005;17:451-66.

32. LaDou J, Castleman B, Frank A, Gochfeld M, Greenberg M, Huff $\mathrm{J}$, et al. The case for a global ban on asbestos. Environ Health Perspect. 2010;118:897-901.

33. Joshi TK, Gupta RK. Asbestos in developing countries: magnitude of risk and its practical implications. Int $\mathrm{J}$ Occup Med Environ Health. 2004;17:179-85. 